\title{
Two Trees Which Are Self-intersecting When Drawn Simultaneously
}

\author{
Markus Geyer $^{1}$, Michael Kaufmann ${ }^{1}$, and Imrich Vrto ${ }^{2, \star}$ \\ 1 Universität Tübingen, WSI für Informatik, Sand 13, \\ 72076 Tübingen, Germany \\ \{mk, geyer\}@informatik.uni-tuebingen.de \\ 2 Institute of Mathematics, Slovak Academy of Sciences, \\ Dúbravská 9, 84104 Bratislava, Slovakia \\ vrto@savba.sk
}

\begin{abstract}
An actual topic in the graph drawing is the question how to draw two edge sets on the same vertex set, the so-called simultaneous drawing of graphs. The goal is to simultaneously find a nice drawing for both of the sets. It has been found out that only restricted classes of planar graphs can be drawn simultaneously using straight lines and without crossings within the same edge set. In this paper, we negatively answer one of the most often posted open questions namely whether any two trees with the same vertex set can be drawn simultaneously crossing-free in a straight line way.
\end{abstract}

\section{Introduction}

Recently, a new direction in the area of the graph drawing has been opened: Simultaneous planar graph drawing [1, 3, 4, 5, 6. Consider a set of objects with two different sets of relations. Such structures arise in many applications, e.g. in software engineering, databases, and social networks. The goal is to draw both underlying graphs on the same set of vertices in the plane using straight lines such that each graph alone is displayed as nicely and readable as possible. In case that both graphs are planar, we require that every graph itself is embedded in a plane way. More formally, given two planar graphs $G_{1}=\left(V, E_{1}\right)$ and $G_{2}=\left(V, E_{2}\right)$, simultaneous drawing of $G_{1}$ and $G_{2}$ is to find their plane straight line drawings $D_{1}$ and $D_{2}$, such that every vertex is mapped to the same point in both $D_{1}$ and $D_{2}$. Brass et al. 1 proved that two paths, two cycles and two caterpillars can always be drawn simultaneously. A caterpillar is such a tree that the graph obtained by deleting the leaves is a path. On the other hand, they constructed 2 outerplanar graphs for which the simultaneous drawing is impossible. Erten and Kobourov [5] found an example of a planar graph and a path that do not allow a simultaneous drawing. The most posted open problem in this area is the question whether two trees can always be drawn simultaneously [1,2,5. In this

\footnotetext{
* Supported by a DFG grant "Graphenzeichnen in der Anwendung" No. K812/8-2 and a VEGA grant No. 2/3164/23.
} 
paper we answer this question in negative. Our counterexample consists of two isomorphic trees of depth 2 .

\section{The Counterexample}

The two trees $T_{1}(n)=\left(V, E_{1}\right)$ and $T_{2}(n)=\left(V, E_{2}\right)$ are given as follows: $T_{1}(n)$ and $T_{2}(n)$ have a common root $r$ with $n$ common children $v_{1}, \ldots, v_{n}$. The parameter $n$ will be determined later. The children $v_{i}, 1 \leq i \leq n$ of $r$ have again children $v_{i j}, 1 \leq i, j \leq n, i \neq j$, s.t. $\left(v_{i}, v_{i j}\right) \in E_{1}$ and $\left(v_{j}, v_{i j}\right) \in E_{2}$. We call the edges in $E_{1} \cap E_{2}$ thick black, in $E_{1} \backslash E_{2}$ thin black and those from $E_{2} \backslash E_{1}$ thin gray. We denote the union of the two trees by $G_{n}$. A straight line drawing of $G_{n}$ is called partially planar if there is no crossing of 2 edges from $E_{1}$ nor crossing of 2 edges from $E_{2}$, which is equivalent to the simultaneous drawing of $T_{1}(n)$ and $T_{2}(n)$. Fig. 1 shows a partially planar drawing of $G_{4}$. Note that this graph class has already been described in [5].

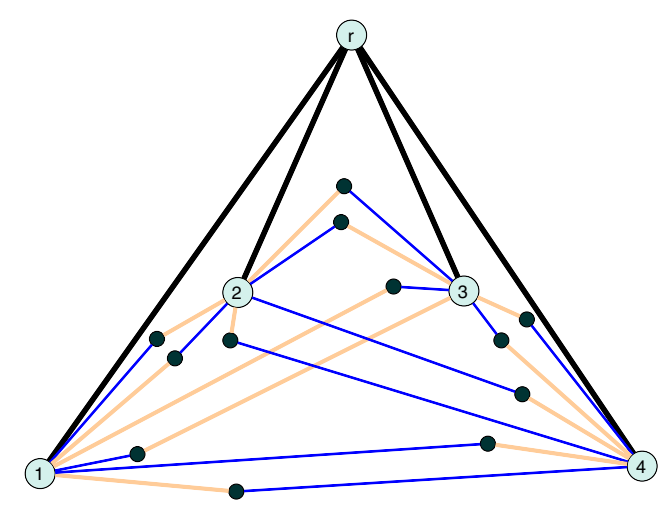

Fig. 1. An example that shows a partially planar drawing of $G_{4}$

Theorem 1. For $n \geq 15$, the simultaneous drawing of $G_{n}$ is self-intersecting.

Proof. Since $G_{15}$ is a subgraph of $G_{n}, n \geq 15$ it is sufficient to prove that any simultaneous drawing for $G_{15}$ is self-intersecting.

Let us assume that there is a partially planar layout $L_{15}$ for $G_{15}$. We consider such a layout $L_{15}$ and derive a contradiction.

The proof proceeds in three steps:

Lemma 1. In any partially planar layout $L_{15}$, there are 8 children of the root such that in the corresponding sublayout $L_{8}$ for the subgraph $G_{8}$ induced by the root $r$, the 8 children and the leafs on the connections between them, the root $r$ lies on the outer face of $L_{8}$.

In the following, we only argue on the layout $L_{8}$ of the 8 children from the previous lemma and derive a contradiction for $L_{8}$. The indexing is done as the 
children appear in clockwise order seen from the root. We also consider our indexing to be counted $(\bmod 8)+1(\operatorname{or}(\bmod 5)+1$ respectively $)$, s.t. after $v_{8}$ (or $v_{5}$ respectively) we have $v_{1}$ again in clockwise order.

Lemma 2. Let $G_{5}$ be any subgraph of the $G_{8}$ induced by the root $r$, by any 5 children $v_{1}, \ldots, v_{5}$ out of the eight children of the root and by the leafs on the connections between these 5 vertices. For all vertices $v_{i}, 1 \leq i \leq 5$, the two 4-gons defined by the straight-line segments $\left(v_{i}, v_{i j}\right),\left(v_{i j}, v_{j}\right),\left(v_{j}, v_{j i}\right)$ and $\left(v_{j i}, v_{i}\right), j=i-2, i+2$ do intersect.

And finally

Lemma 3. For any layout $L_{8}$ of $G_{8}$ as defined above there is a vertex $v_{i}$, $1 \leq i \leq 8$, such that the two 4-gons defined by the straight-line segments $\left(v_{i}, v_{i j}\right),\left(v_{i j}, v_{j}\right),\left(v_{j}, v_{j i}\right)$ and $\left(v_{j i}, v_{i}\right), j=i-2, i+2$ do not intersect.

Lemma 3 is obviously in contradiction to Lemma 2, therefore no such layout could exist.

In the following section, we provide the proofs of the three lemmata plus all the necessary definitions and useful observations concerning the structures of the layout.

\section{The Proofs}

\subsection{Identifying an Appropriate Subgraph}

Lemma 4. In any partially planar layout $L_{15}$, there are 8 children of the root such that in the corresponding sublayout $L_{8}$ for the subgraph $G_{8}$ induced by the root $r$, the 8 children and the leafs on the connections between them, the root $r$ lies on the outer face of $L_{8}$.

Proof. Let $L_{15}$ be a partially planar layout of graph $G_{15}$. Let $C=\left\{v_{1}, \ldots, v_{1} 5\right\}$ be the children of the root in clockwise order. We identify two children $v_{i}$ and $v_{j}$ such that the polygon formed by $\left(r, v_{i}\right),\left(v_{i}, v_{i j}\right),\left(v_{i j}, v_{j}\right)$ and $\left(v_{j}, r\right)$ encloses a maximal number of children of the root. Note that $v_{i}$ and $v_{j}$ may not be unique.

Let $S$ be the set of children within the polygon with $|S|=k$. It is easy to see that for the whole subgraph $G_{k}$ induced by the root $r$, the children in $S$ and the leafs on the connections between them, the root $r$ lies on the outer face. If $k \geq 8$ we are done and can arbitrarily choose 8 of the children in $S$ to form our subgraph $G_{8}$. If $k<8$, we consider the set $C \backslash S$ of size $l \geq 8$. By the choice of $i$ and $j$ we know that all but one of the connections within $C \backslash S$ lie on the 'same' side of the root such that removing only the two segments $\left(v_{i}, v_{i j}\right)$ and $\left(v_{i j}, v_{j}\right)$ will bring $r$ to the outer face of the layout for the subgraph $G_{l}$ induced by the root $r$, the set $C \backslash S$ and the corresponding leafs. For this case, any subset of $C \backslash S$ of size 8 will provide us the desired subgraph $G_{8}$. 


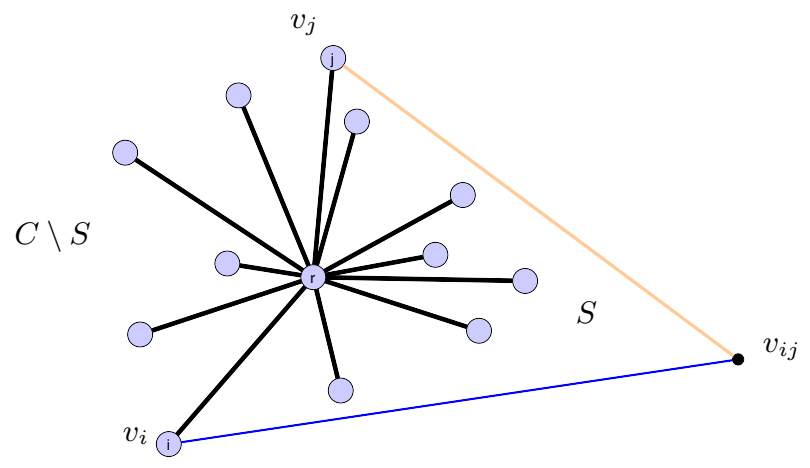

Fig. 2. An example for the choice of $v_{i}$ and $v_{j}$

\subsection{Structural Characterizations}

After having identified a partially planar layout $L$ for a $G_{8}$ such that the root lies at the outer face, we start with some characterizations to prepare the proof of the main lemma.

Take any 5 vertices $v_{1}, \ldots, v_{5}$ out of those 8 children in clockwise order. Consider the corresponding graph $G_{5}$ induced by the root, by $v_{1}, \ldots, v_{5}$ and by $v_{i j}, 1 \leq i, j \leq 5, i \neq j$ and its corresponding layout $L_{5}$. Clearly $L_{5}$ is partially planar.

- Note that each leaf $v_{i j}$ has actually two adjacent vertices, namely $v_{i}$ and $v_{j}$ by a gray edge and by a thin black edge. We can also view each pair of vertices $v_{i}$ and $v_{j}$ as being connected by two 2 -segment polylines, where one is colored gray/thin black and the other thin black/gray.

- The two connecting 2-segments between each pair $v_{i}$ and $v_{j}$ form a 4-gon $P_{i j}$. It is clear that the participating four segments do not cross.

- None of the 5 vertices lies inside of the $P_{i j}$ and none is enclosed by a sequence of $P_{i j}$ 's. This means that each of them lie on the outer face of the planar subdivision formed by the edges $v_{i} v_{i j}$ and $v_{j} v_{i j}$. This is enforced by the black edges from the root to the vertices $v_{i}$ and by our condition that none of the $P_{i j}$ or a sequence of those enclose the root.

- We say that polygons $P_{i j}$ and $P_{l k}$ are intersecting if a segment of $P_{i j}$ crosses a segment of $P_{l k}$. Otherwise they are independent.

- Note that a vertex $v_{i}$ can only have two neighboring vertices $v_{j}, v_{k}$. That are vertices, such that the polygons $P_{i j}$ and $P_{i k}$ are not separating any two vertices. That means the polygons $P_{i j}$ and $P_{i k}$ can be assumed to be independent from each other and from the remaining 4-gons. We also assume from now on, that our numbering reflects this neighbor property and is in clockwise order, e.g. $v_{i}$ is neighbor to $v_{i+1}$ for $i \in\{1, \ldots, 4\}$ and $v_{5}$ is neighbor to $v_{1}$.

- The following three configurations for two intersecting polygons $P_{i j}$ and $P_{l k}$ with $i<l<j<k$ are the basics. 
Configuration 1: The two leaves incident to $P_{i j}$ lie inside the polygon $P_{l k}$ and the two leaves incident to $P_{l k}$ lie inside the polygon $P_{i j}$.

Configuration 2: Exactly one leaf of $P_{i j}$ lies inside the polygon $P_{l k}$ and exactly one leaf of $P_{l k}$ lies inside the polygon $P_{i j}$.

Configuration 3: The two leaves incident to $P_{i j}$ lie outside the polygon $P_{l k}$ and the two leaves incident to $P_{l k}$ lie outside the polygon $P_{i j}$. See Fig. 3 .

- Note that for each polygon the colors can be switched.
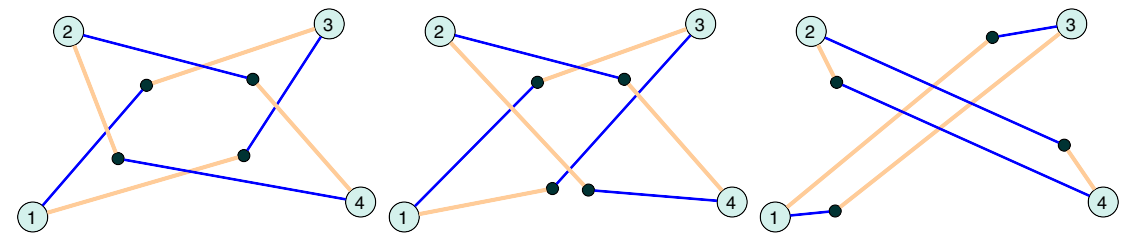

Fig. 3. Configuration 1,2 and 3

Next we state the main structural lemma, which is identical to our former Lemma 2,

Lemma 5. In any partially planar drawing of $G_{5}$, for each $v_{i}, 1 \leq i \leq 5$, there are two 2-segment connections to $v_{i+2}$ and to $v_{i-2}$ that cross.

Proof. Assume that there is a vertex $v_{i}$ contradicting the claim. By renumbering, we assume that $v_{i}=v_{1}$. This means that the polygons $P_{13}$ and $P_{14}$ are independent.

In what follows we perform a case analysis. On the top level we distinguish two different clockwise orders of the four incident segments of the two polygons attached to $v_{1}$ :

A) thin black, gray, gray, thin black and

B) thin black, gray, thin black, gray.

Clearly, both polylines of $P_{14}$ separate $v_{3}$ from $v_{5}$. So $P_{14}$ and $P_{35}$ are intersecting.

Then we discuss for each of A) and B) the Configurations 1,2 and 3, described above, for mutual positions of $P_{14}$ and $P_{35}$.

And finally, for each of the previous cases we have two subcases:

a) Polygons $P_{31}$ and $P_{35}$ are independent.

b) Polygons $P_{31}$ and $P_{35}$ are intersecting.

Vertex $v_{2}$ is on the convex hull between $v_{1}$ and $v_{3}$. It now has to be connected by two bicolored curves to $v_{4}$ as well as to $v_{5}$. We describe the possible route of the four paths by the sequence of segments that have to be crossed. Fortunately, this sequence is almost unique.

Before we dive into the case analysis, we formulate some conditions for the solvability: 
- incident-segments-condition: Two straight line segments adjacent to the same vertex obviously cannot cross.

- straightness-condition: Two straight line segments cross at most once.

- one-two-condition: Consider a drawing where segments $s$ and $s^{\prime}$ are adjacent to vertex $v, s^{\prime}$ forms a double-segment with $s^{\prime \prime}$ and in addition $s^{\prime \prime}$ crosses $s$. W.l.o.g. we can assume that there is no such configuration, since any such configuration can easily be redrawn into a configuration where the crossing has been removed.

We assume that the one-two-condition is obeyed in the solution and we always construct a contradiction to the incident-segments condition:

Case A1a: In the Fig. 4(a) consider the two dashed polygonal lines connecting the vertices $v_{2}$ to $v_{4}$ and $v_{5}$ respectively. They indicate the potential route of the corresponding double-segments. Clearly the observation holds that at least one of the two curves have to change its color next to vertex $v_{2}$ or within the first polygon $P_{13}$. If the gray curve changes its color then it follows the thin black curve to $v_{5}$ and completely indicates the topological route of the gray-thin black curve from $v_{2}$ to $v_{5}$. Clearly, this is a contradiction to the incident-segment-condition since the last segment of the gray-thin black curve from $v_{2}$ to $v_{5}$ intersects the last segment of the thin black-gray double segment from $v_{3}$ to $v_{5}$. Similarly, if the thin black curve changes its color next to $v_{2}$ it follows the gray curve to $v_{4}$. As before, we achieve a contradiction to the incident-segment condition since the last segment of the thin black-gray curve from $v_{2}$ to $v_{4}$ intersects the last segment of the gray-thin black double segment from $v_{2}$ to $v_{4}$.

Case A1b,A2b: Since the one-two-condition is violated by the thin black-gray double segment between $v_{3}$ and $v_{1}$ and the first thin black segment between $v_{3}$ and $v_{5}$, we can safely assume that these cases does not occur.

Case A2a: Analogously as in case A1a, we argue that one of the two curves from Fig. 4(a) has to change colors next to $v_{2}$. It therefore indicates one of the routes from $v_{2}$ to $v_{4}$ or $v_{5}$. As in the case A1a, we get a contradiction to the incident-segment condition.

Case A3a: (See Fig. 4(e), The two curves indicate the similarity to case A1a. One of the curves has to change its color close to $v_{2}$ and therefore it produces a violation of the incident-segments-condition.

Case A3b: (See Fig. 4(f) As before, the curves and the color changing close to $v_{2}$ lead to a violation of the incident-segments condition.

Next, we will consider the case B, where we assume that the clockwise order of the edges incident to $v_{1}$ is thin black, gray, thin black, gray. The arguments are along the same lines as in case A, but for completeness we consider all the cases:

Case B1a: In Fig. 5(a) we show the two canonical curves one of which has to change its color near $v_{2}$ and then follow the other one. Clearly, the same kind of contradiction to the incident-segments condition occurs as in case A1a. 


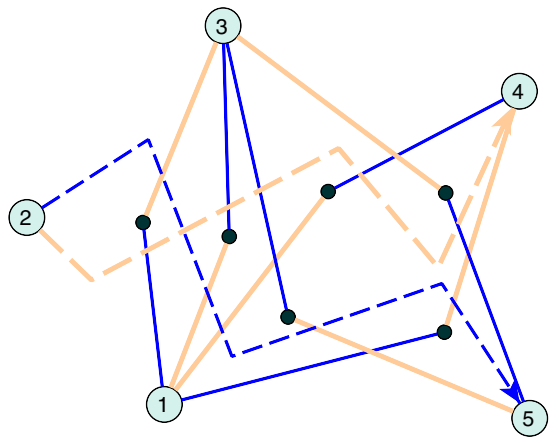

(a) Case A1a enhanced by two potential routes

(2)

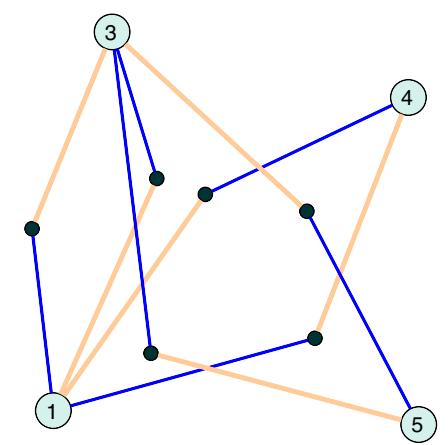

(c) The case A1b: $P_{31}$ and $P_{35}$ are intersecting

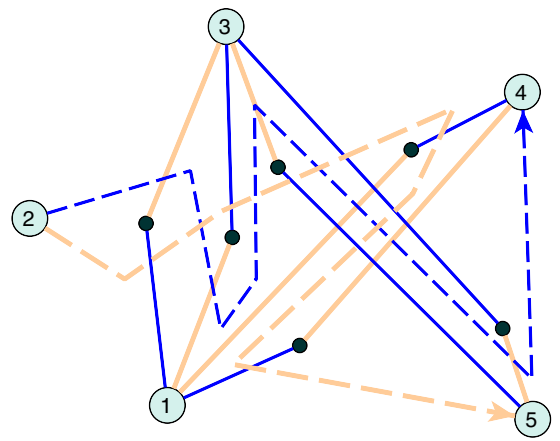

(e) Case A3a enhanced by two potential routes

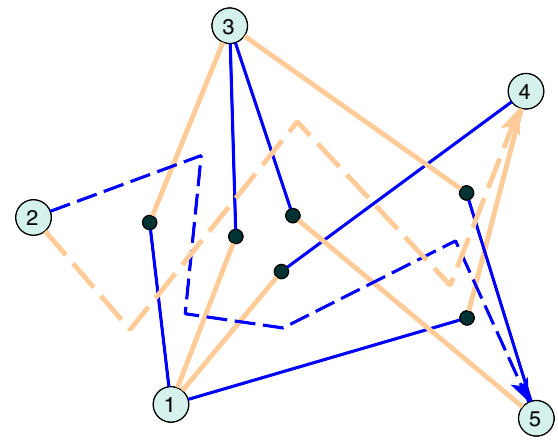

(b) The case A2a enhanced by two potential routes

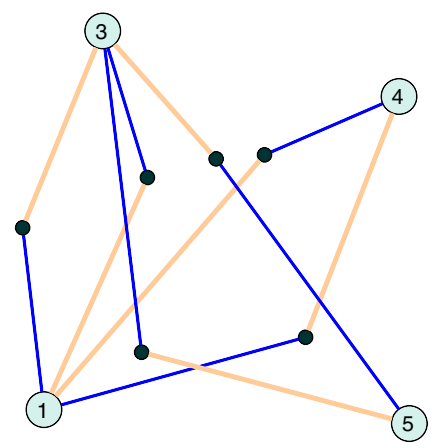

(d) The case A2b: $P_{31}$ and $P_{35}$ are independent

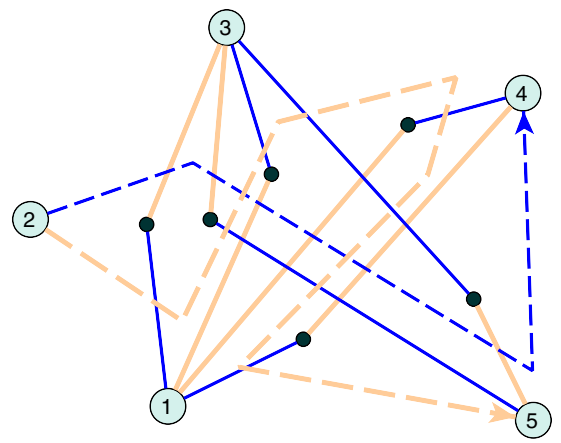

(f) Case A3b enhanced by two potential routes

Fig. 4. The different cases for the clockwise ordering thin black, gray, gray, thin black

Case B1b: This case cannot occur at all since there is a crossing of segments of the same color.

Case B2a: (See Fig. 5(c)]) As before, the two curves that uniquely indicate the routes induce at least one contradiction to the incident-segments-condition. 


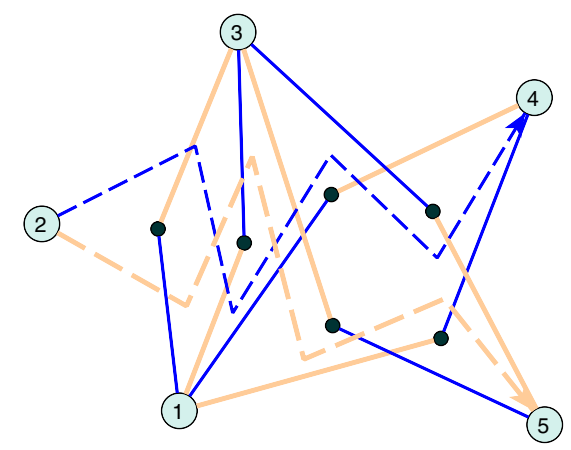

(a) Case B1a enhanced by two potential routes

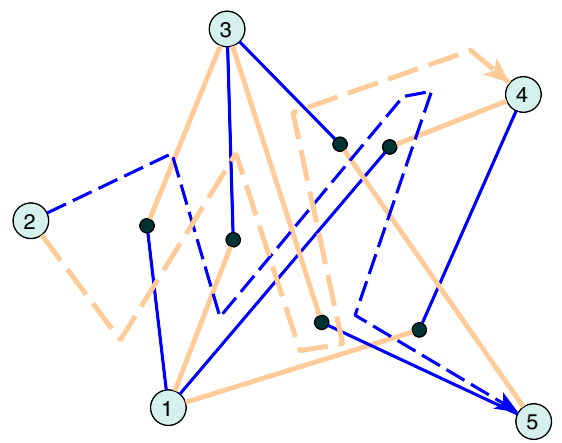

(c) Case B2a enhanced by two potential routes

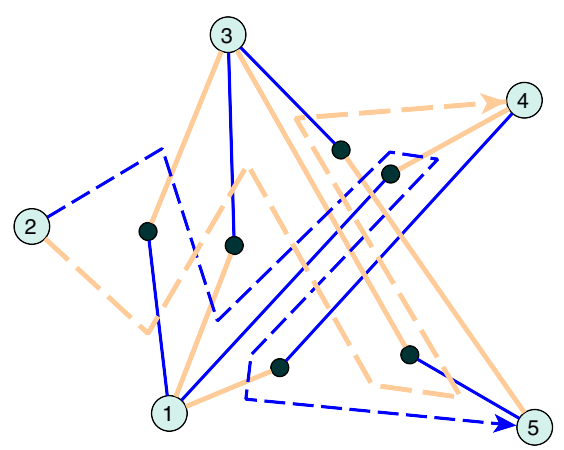

(e) Case B3a enhanced by two potential routes
(2)

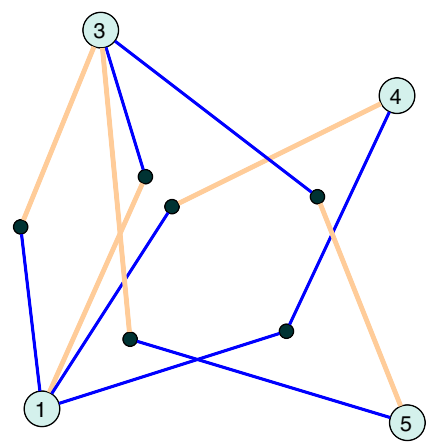

(b) The case B1b

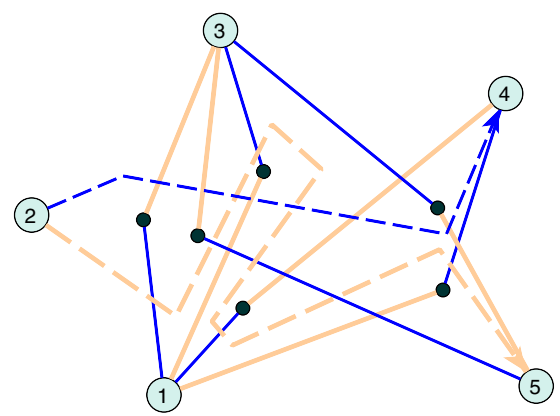

(d) Case B2b enhanced by two potential routes

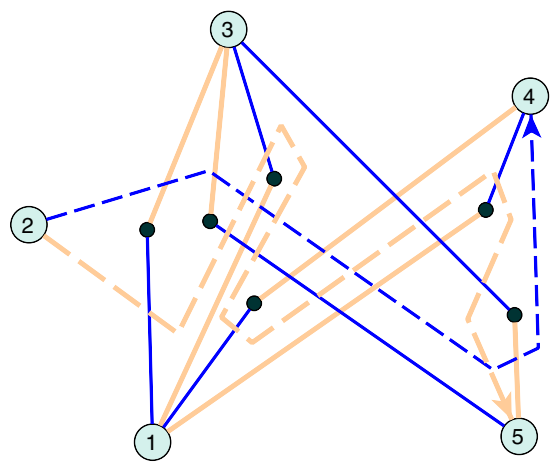

(f) Case B3b enhanced by two potential routes

Fig. 5. The different cases for the clockwise ordering thin black, gray, thin black, gray

Case B2b: (See Fig. 5(d).) Similar to the case B2a. Although the thin black curve looks promising it violates the incident-segments-condition since it crosses the first gray segment of the double segment from $v_{4}$ to $v_{1}$. 
Case B3a: (See Fig. 5(e) The snakelike curves immediately lead to a contradiction to the incident-segments-condition.

Case B3b: (See Fig. 5(f), Analogously to the case B3a. This concludes the proof of the main lemma.

\subsection{The Final Argument}

With the next lemma we state a property for any layout of $G_{8}$, which is in direct contradiction to a property that has been shown in Lemma 5 .

Lemma 6. For any layout $L_{8}$ of $G_{8}$ as defined above there is a vertex $v_{i}, 1 \leq i \leq$ 8 , such that the two 4-gons defined by the straight-line segments $\left(v_{i}, v_{i j}\right),\left(v_{i j}, v_{j}\right)$, $\left(v_{j}, v_{j i}\right)$ and $\left(v_{j i}, v_{i}\right), j=i-2, i+2(\bmod 8)$ do not intersect.

Proof. Assume the 8 children are numbered in clockwise order, see Fig. 6. By Lemma 5 the polygons $P_{13}$ and $P_{17}$ must intersect. The one-two-condition implies that both polygons lie in the halfplane given by the line $v_{3}-v_{7}$ and the vertex $v_{1}$. Symmetrically, the polygons $P_{35}$ and $P_{57}$ lie in the other halfplane. Hence the polygons $P_{13}$ and $P_{35}$ do not intersect.

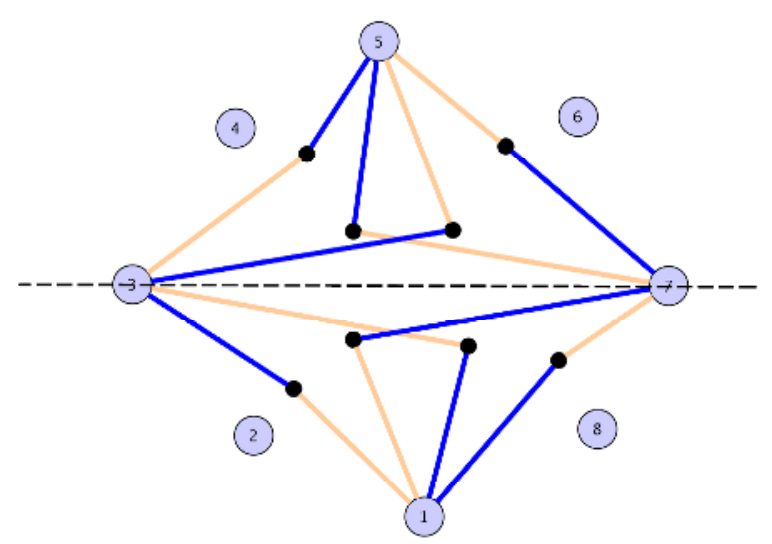

Fig. 6. Polygons $P_{31}$ and $P_{35}$ do not intersect

\section{Conclusion}

We gave an example of a class of tree pairs that are self-intersecting when drawn simultaneously, but unfortunately the parameter $n$ implies a number of $n^{2}+1$ vertices, our smallest counterexample has size 226 . We are optimistic that by more refined arguments this can be improved to $n=8$ or even $n=7$.

Another open question is to give a pair of edge-disjoint trees that are selfintersecting when drawn simultaneously. The class $G_{n}$ can easily be generalized to contain two edge-disjoint trees but our argument for the self-intersection 
heavily relied on the straight-line edges that are contained in both trees. Finally, it remains an obvious task to generalize these arguments (or find new one) to prove self-intersection for simpler classes of graphs like a tree and a caterpillar.

\section{Acknowledgments}

The second author wishes to thank Stephen Kobourov for giving him enough motivation for the final push on the paper during the Dagstuhl-Seminar No. 5191 on Graph Drawing.

\section{References}

1. Brass, P., Cenek, E., Duncan, A., Efrat, A., Erten, C., Ismailescu, D., Kobourov, S., Lubiw, A., Mitchell, J.S.B.: On Simultaneous Planar Graph Embeddings. In: Dehne F., Sack, J., Snid, M. (eds.): Workshop on Algorithms and Data Structures, Lecture Notes in Computer Science, Vol. 2748. Springer, Berlin (2002) 243-255

2. Duncan, C.A., Eppstein, D., Kobourov, S.G.: The Geometric Thickness of Low Degree Graphs. In: Boissonant, J.-D., Snoeyink, J. (eds.): 23rd Annual Symp. on Computational geometry. ACM Press, New York (2004) 340-346

3. Erten, C., Kobourov, S.G.: Simultaneous Embedding of a Planar Graph and its Dual on the Grid. In: Bose, P., Morin, P. (eds.): 13th Intl. Symp. on Algorithms \& Computation. Lecture Notes in Computer Science, Vol. 2518. Springer, Berlin (2002) 575-587

4. Erten, C., Kobourov, S.G., Le, V., Navabi, A.: Simultaneous Graph Drawing: Layout Algorithms and Visualization Schemes. In: Liotta, G. (ed.): 11th Intl. Symp. on Graph Drawing. Lecture Notes in Computer Science, Vol. 2912. Springer, Berlin (2003) 437-449

5. Erten, C., Kobourov, S.G.: Simultaneous Embedding of Planar Graphs with Few Bends. In: Pach, J. (ed.): 12th Intl. Symp. on Graph Drawing. Lecture Notes in Computer Science, Vol. 3383. Springer, Berlin (2004) 195-205

6. Kobourov, S.G., Pitta, C.: An Interactive Multi-User System for Simultaneous Graph Drawing. In: Pach, J. (ed.): 12th Intl. Symposium on Graph Drawing. Lecture Notes in Computer Science, Vol. 3383. Springer, Berlin (2004) 492-501 